\title{
ON SOME SMALL VARIETIES OF DISTRIBUTIVE OCKHAM ALGEBRAS
}

\author{
by R. BEAZER
}

(Received 7 January, 1983)

1. Introduction. J. Berman [2] initiated the study of a variety $\mathscr{K}$ of bounded distributive lattices endowed with a dual homomorphic operation paying particular attention to certain subvarieties $\mathscr{K}_{m, n}$. Subsequently, A. Urquhart [8] named the algebras in $\mathscr{K}$ distributive Ockham algebras, and developed a duality theory, based on $\mathrm{H}$. A. Priestley's order-topological duality for bounded distributive lattices [6], [7]. Amongst other things, Urquhart described the ordered spaces dual to the subdirectly irreducible algebras in $\mathscr{K}$. This work was developed further still by M. S. Goldberg in his thesis and the paper [5]. Recently, T. S. Blyth and J. C. Varlet [3], in abstracting de Morgan and Stone algebras, studied a subvariety MS of the variety $\mathscr{K}_{1,1}$. The main result in [3] is that there are, up to isomorphism, nine subdirectly irreducible algebras in MS and their Hasse diagrams are exhibited. The methods employed in [3] are purely algebraic and can be generalized to show that, up to isomorphism, there are twenty subdirectly irreducible algebras in $\mathscr{K}_{1,1}$. In section 3 of this paper, we take a short cut to this result by utilizing the results of Urquhart and Goldberg. Our basic method is simple: the results of Goldberg [5] are applied to $\mathscr{K}_{1,1}$ to produce a certain eight-element algebra $B_{1}$ in $\mathscr{K}_{1,1}$, whose lattice reduct is Boolean and whose subalgebras are, up to isomorphism, precisely the subdirectly irreducibles in $\mathscr{K}_{1,1}$. We then pick out of the list of twenty such algebras those belonging to the variety MS. In section 4, we sketch a purely algebraic proof along the lines followed by Blyth and Varlet in [3].

2. Preliminaries. A distributive Ockham algebra is an algebra $\left\langle L, \vee, \wedge,{ }^{0}, 0,1\right\rangle$ of type $\langle 2,2,1,0,0\rangle$ such that $\langle L, \vee, \wedge, 0,1\rangle$ is a bounded distributive lattice and ${ }^{\circ}$ is a unary operation defined on $L$ such that, for all $x, y \in L$,

$$
(x \wedge y)^{0}=x^{0} \vee y^{0}, \quad(x \vee y)^{0}=x^{0} \wedge y^{0}, \quad 0^{0}=1, \quad 1^{0}=0 .
$$

The class of all distributive Ockham algebras is a variety, henceforth denoted by $\mathbf{0}$, and the subvariety of 0 defined by the identity $x^{0}=x^{000}$ is the aforementioned variety $\mathscr{K}_{1,1}$.

An $M S$ algebra is an algebra $\left\langle L, \vee, \wedge,{ }^{0}, 0,1\right\rangle$ of type $\langle 2,2,1,0,0\rangle$ such that, for all $x, y \in L$,

$$
x \wedge x^{00}=x, \quad(x \wedge y)^{0}=x^{0} \vee y^{0}, \quad 1^{0}=0 .
$$

The variety MS of MS algebras is shown in [3] to be a proper subvariety of $\mathscr{K}_{1,1}$.

For all unexplained lattice theoretic and universal algebraic terminology and notation we refer the reader to [1]. Throughout, we assume familiarity with H. A. Priestley's duality for bounded distributive lattices, at least in the finite case, and outline just enough of the duality for the class of finite Ockham algebras, to achieve our aims. For the general duality theory of distributive Ockham algebras we refer the reader to [5] and [8].

Glasgow Math. J. 25 (1984) 175-181. 
If $g$ is an order reversing map from a finite poset $X$ into itself then the pair $(X ; g)$, sometimes compressed to $X$, is called an Ockham space. Any finite Ockham space $(X ; g)$ gives rise to a finite distributive Ockham algebra, called the dual algebra of $X$ and denoted $O(X)$. Indeed, a dual endomorphism ${ }^{\circ}$ can be defined on the distributive lattice of order ideals of $X$ by $I^{0}=X \backslash g^{-1}(I)$, for each order ideal $I$ of $X$. Moreover, given any finite $A \in \mathbf{0}$, the pair $(P(A) ; g)$, where $P(A)$ is the poset of prime ideals of $A$ and $g: P(A) \rightarrow P(A)$ is defined by $g(P)=\left\{a \in A ; a^{0} \notin P\right\}$ is a finite Ockham space, called the dual space of $A$ and denoted by $\mathscr{S}(A)$, and $A$ is isomorphic to its second dual.

3. Subdirectly irreducibles in $\mathscr{K}_{1,1}$ and MS. For integers $m, n$ satisfying $m>n \geq 0$, $\mathbf{P}_{m, n}$ will denote the subclass of $\boldsymbol{0}$ consisting of those algebras $A$ satisfying the identity $x^{m}=x^{n}$, where elements $a^{m} \in A$ are defined by

$$
a^{0}=a, \quad a^{k+1}=\left(a^{k}\right)^{0} \quad \text { whenever } \quad k \geqslant 0 .
$$

The classes $\mathbf{P}_{m, n}$ are shown in [5] and [8] to play a fundamental role and the aforementioned subvarieties $\mathscr{K}_{m, n}$ of $\mathbf{0}$ studied by J. Berman [2] correspond to the classes $\mathbf{P}_{2 m+n, n}$. Of particular relevance here: $\mathscr{K}_{1,1}=\mathbf{P}_{3,1}$. The Ockham spaces $(X ; g)$ which are dual spaces of algebras in $\mathbf{P}_{m, n}$ are precisely those with $g^{m}=g^{n}$ and it is straightforward to show that an Ockham space $(X ; g)$ is the dual space of an MS-algebra if and only if $g^{2}(x) \leqslant x$, for all $x \in X$.

For integers $m, n$ satisfying $m>n \geq 0$, let $\mathbf{m}_{n}$ denote the pair $\left(\mathbf{m}, \gamma_{n}\right)$, where $\mathbf{m}=$ $\{0,1, \ldots, m-1\}$ is endowed with the discrete order and $\gamma_{n}: \mathbf{m} \rightarrow \mathbf{m}$ is defined by

and

$$
\gamma_{n}(k)=k+1 \text {, whenever } 0 \leqslant k<m-1 \text {, }
$$

$$
\gamma_{n}(m-1)=n \text {. }
$$

Observe that, since the order on $\mathbf{m}_{n}$ is discrete, $L_{m, n}=0\left(\mathbf{m}_{n}\right)$ has a Boolean lattice reduct and so, according to [5], the subdirectly irreducible algebras in $\mathbf{P}_{m, n}$ are precisely the subalgebras of $L_{m, n}$. It is a simple matter to see that $L_{3,1}$ is the power set lattice of the set $\{0,1,2\}$ endowed with the unary operation ${ }^{0}$ given by

$$
\begin{gathered}
\phi^{0}=\{0\}^{0}=\{0,1,2\}, \\
\{0,1,2\}^{0}=\{1,2\}^{0}=\phi, \\
\{0,1\}^{0}=\{1\}^{0}=\{1\}, \\
\{2\}^{0}=\{0,2\}^{0}=\{0,2\} .
\end{gathered}
$$

The Hasse diagram of $L_{3,1}$ is subsequently labelled $B_{1}$. If $H$ is the Hasse diagram of an algebra in $\mathbf{O}$ then by the dual of $H$ we will mean the diagram $\breve{H}$ obtained by inverting $H$. In this terminology we have the following result.

THEOREM 1. The variety $\mathscr{K}_{1,1}$ has, up to isomorphism, twenty subdirectly irreducible algebras and they are described by the Hasse diagrams in Figure 1 together with their duals.

Eliminating, by inspection, those of the twenty algebras having an element $x$ such that $x \notin x^{00}$, we have the following result. 

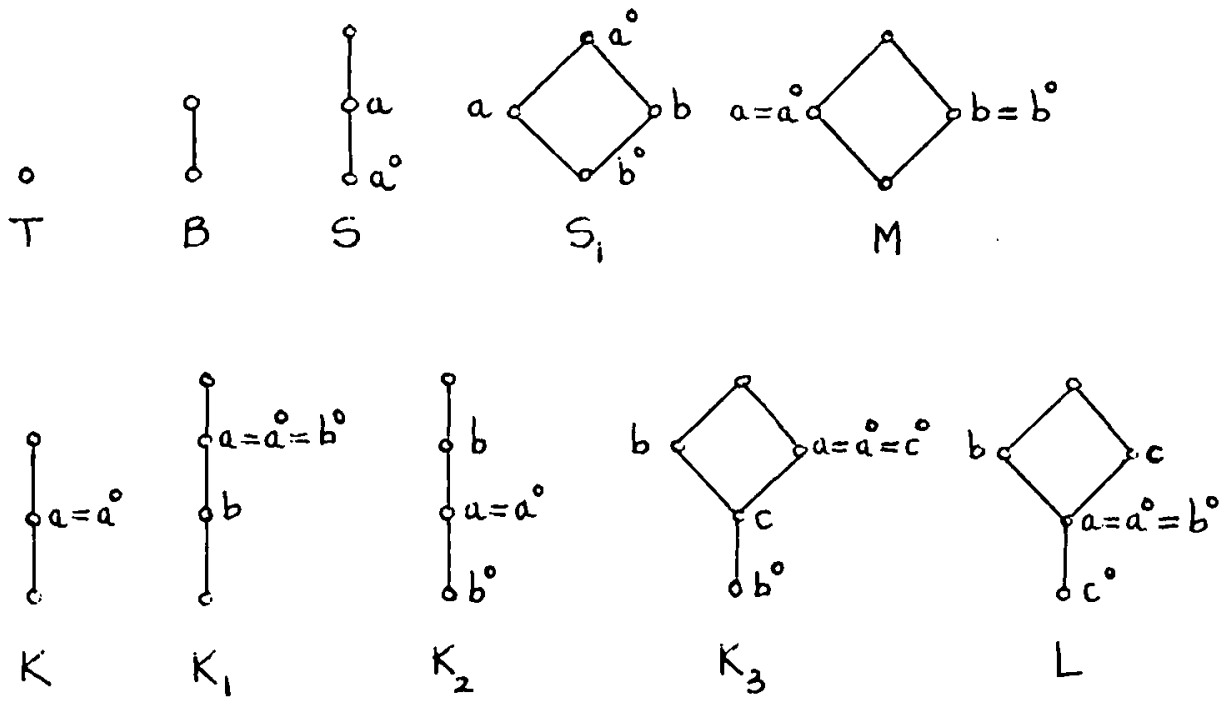

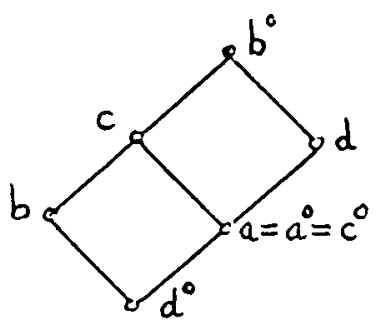

$N$

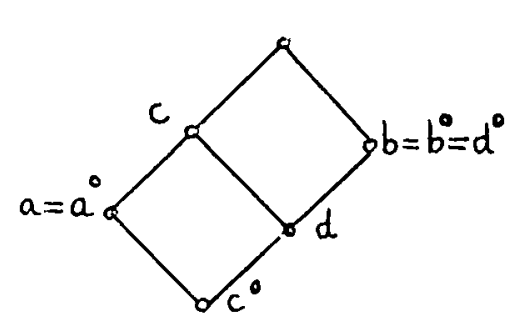

$M$

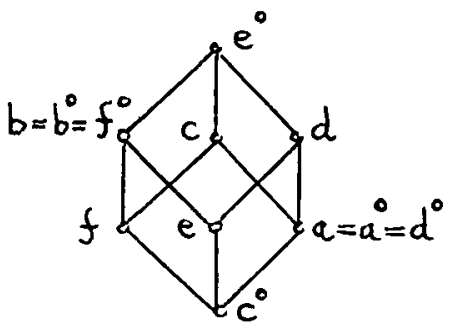

$B$

Figure 1

COROllary 2. ([3]) The variety MS has, up to isomorphism, nine subdirectly irreducible algebras; namely

$$
T, B, S, M, K, K_{1}, K_{2}, K_{3}, M_{1} \text {. }
$$

4. An algebraic approach. Of course, the proof of Theorem 1 presupposes some knowledge of order-topological duality theory. In this section, we sketch an alternative, purely algebraic, proof by generalizing the ideas and methods employed by T. S. Blyth and J. C. Varlet in [3]. We do this not just for comparative purposes but because other factors arise during the discussion which, besides shedding more light on the structure of the subdirectly irreducibles in $\mathscr{K}_{1,1}$, are of independent interest and useful in other directions.

We begin with a result which generalizes from MS to $\mathbf{0}$ a theorem in [3]. The proof is 
substantially different from the corresponding one in [3] for MS in that it does not require the description of principal congruences in $\mathbf{0}$ due to $\mathrm{J}$. Berman [2].

Let $I$ be an ideal of $L \in \mathbf{0}$. For each integer $m<\boldsymbol{\omega}$, define

$$
I_{2 m}=\left\{x \in L ; x \leqslant i^{2 m}, \text { for some } i \in I\right\}
$$

and

$$
I^{2 m+1}=\left\{x \in L ; x \geqslant i^{2 m+1} \text {, for some } i \in I\right\} .
$$

Observe that $I_{2 m}$ is an ideal and $I^{2 m+1}$ is a filter of $L$. Now, let

$$
I_{00}=\bigvee_{m<\omega} I_{2 m} \text { and } I^{0}=\bigvee_{m<\omega} I^{2 m+1},
$$

where the first join is taken in the ideal lattice of $L$ and the second is taken in the filter lattice of $L$.

THEOREM 3. If $I$ is an ideal of $L \in \mathbf{0}$ and $\Theta(I)$ is the smallest congruence of $L$ collapsing $I$, then

$$
x \equiv y(\Theta(I)) \Leftrightarrow(x \vee i) \wedge j=(y \vee i) \wedge j,
$$

for some $i \in I_{00}$ and some $j \in I^{0}$.

Proof. Let $\theta_{I}$ denote the relation defined on $L$ by the condition above. By the distributivity of $L, \theta_{I}$ is a lattice congruence. Moreover, if $(x \vee i) \wedge j=(y \vee i) \wedge j$, for some $i \in I_{00}$ and $j \in I^{0}$, then, operating on both sides by ${ }^{0}$ and using distributivity, we have

$$
\left(x^{0} \vee j^{0}\right) \wedge\left(i^{0} \vee j^{0}\right)=\left(y^{0} \vee j^{0}\right) \wedge\left(i^{0} \vee j^{0}\right) \text {. }
$$

We claim that $j^{0} \in I_{00}$ and $i^{0} \in I^{0}$. Indeed, since $j \in I^{0}$, there are positive integers $m_{k}$ and elements $a_{k} \in I^{2 m_{k}+1}, 1 \leqslant k \leqslant r$, such that $j=a_{1} \wedge \ldots \wedge a_{r}$. However, $a_{k} \geqslant i_{k}^{2 m_{k}+1}$, for some $i_{k} \in I$, so that $a_{k}^{0} \leqslant i_{k}^{2\left(m_{k}+1\right)}$ and therefore $a_{k}^{0} \in I_{2\left(m_{k}+1\right)}$. Thus, $j^{0}=a_{1}^{0} \vee \ldots \vee a_{r}^{0} \in I_{00}$. Similarly, $i^{0} \in I^{0}$ and so $i^{0} \vee j^{0} \in I^{0}$, since $I^{0}$ is a filter of $L$. It follows, now, that $x^{0} \equiv y^{0}\left(\theta_{I}\right)$. We conclude that $\theta_{I}$ preserves the operation ${ }^{0}$ and so is a congruence of $L$. Obviously, $I \subseteq[0] \theta_{I}$, so that $\theta_{I}$ collapses $I$, and it remains only to show that it is the smallest such congruence of $L$. Let $\theta$ be any congruence of $L$ collapsing $I$. We claim that $\theta$ also collapses $I_{00}$ and $I^{0}$. Indeed, if $x \in I_{00}$ then there are positive integers $m_{k}$ and elements $b_{k} \in I_{2 m_{k}}, 1 \leqslant k \leqslant s$, such that $x=b_{1} \vee \ldots \vee b_{s}$. However, $b_{k} \leqslant i_{k}^{2 m_{k}}$ for some $i_{k} \in I$, so that $x \leqslant i_{1}^{2 m_{1}} \vee \ldots \vee i_{s}^{2 m_{s}} \equiv 0(\theta)$, since $\theta$ collapses $I$. Therefore, $x \equiv 0(\theta)$ and we conclude that $I_{00} \subseteq[0] \theta$. Similarly, $I^{0} \subseteq[1] \theta$ and so $\theta$ collapses $I^{0}$. Finally, observe that if $x \equiv y\left(\theta_{l}\right)$, so that there is an $i \in I_{00}$ and $j \in I^{0}$ such that $(x \vee i) \wedge j=(y \vee i) \wedge j$, then $x \equiv(x \vee i) \wedge j(\theta)=$ $(y \vee i) \wedge j \equiv y(\theta)$, since $\theta$ collapses $I_{00}$ and $I^{0}$. Thus, $\theta_{I} \leqslant \theta$.

Corollary 4. If $I$ is an ideal of $L \in \mathscr{K}_{1,1}$ then

$$
x \equiv y(\theta(I)) \Leftrightarrow(x \vee i) \wedge j=(y \vee i) \wedge j,
$$

for some $i \in I \vee I_{2}$ and some $j \in I^{1}$. 
The following lemma generalizes to $\mathscr{K}_{1,1}$ results of T. S. Blyth and J. C. Varlet for MS: its proof requires Corollary 4 and is along the lines of their proof of the corresponding result for MS.

LEMMA 5. Let $L$ be a subdirectly irreducible algebra in $\mathscr{K}_{1,1}$. Then, for all $a \in L$,

(i) $a^{0}$ and $a^{00}$ are comparable,

(ii) $a^{00}>a^{0} \Rightarrow a^{0}=0$.

Proof. (i) Let $a \in L$ and let $\theta_{a}=\theta_{\text {lat }}\left(a^{00}, 1\right) \wedge \theta_{\text {lat }}\left(0, a^{0}\right)$, where $\theta_{\text {lat }}(x, y)$ denotes the principal lattice congruence of $L$ collapsing the pair $x, y$ in $L$, so that $\theta_{a}=\theta_{\text {lat }}\left(a^{0} \wedge a^{00}, a^{0}\right)$. Then, using the distributivity of $L$, the well known description of principal lattice congruences of distributive lattices and the fact that $a^{0}=a^{000}$, it is easy to show that $\theta_{a}$ is a congruence of $L$ and $\theta_{a} \wedge \theta_{a^{0}}=\omega$. It follows that either $\theta_{a}=\omega$, in which case $a^{0} \leqslant a^{00}$, or $\theta_{a^{0}}=\omega$, in which case $a^{00} \leqslant a^{000}=a^{0}$, since $L$ is subdirectly irreducible.

(ii) If $a^{00}>a^{0}>0$ then $\theta\left(0, a^{0}\right) \neq \omega$ and $\theta_{a^{0}}=\theta_{\text {lat }}\left(a^{00} \wedge a^{000}, a^{00}\right)=\theta_{\text {lat }}\left(a^{00} \wedge a^{0}, a^{00}\right)=$ $\theta_{\text {lat }}\left(a^{0}, a^{00}\right)$ so that $\theta_{a^{0}} \neq \omega$. However, $\theta_{a^{\circ}} \wedge \theta\left(0, a^{0}\right)=\omega$. Indeed, if $x \equiv y\left(\theta_{a^{\circ}} \wedge \theta\left(0, a^{0}\right)\right)$ then $x \wedge a^{0}=y \wedge a^{0}, x \vee a^{00}=y \vee a^{00}$ and, on taking $I=\left(a^{0}\right]$ in Corollary 4,

$$
(x \vee i) \wedge j=(y \vee i) \wedge j, \text { for some } i \in\left(a^{0}\right] \vee\left(a^{0}\right]_{2}, \quad j \in\left(a^{0}\right]^{1} .
$$

But $\left(a^{0}\right]_{2}=\left(a^{0}\right]$, since $a^{0}=a^{000}$, so that $i \leqslant a^{0}$ and, therefore, $x \wedge i=y \wedge i$. Also, $\left(a^{0}\right]^{1}=$ $\left[a^{00}\right)$ so that $j \geqslant a^{00}$ and therefore $x \vee j=y \vee j$ which, in conjunction with the equation $(x \vee i) \wedge j=(y \vee i) \wedge j$ and the distributivity of $L$, yields $x \vee i=y \vee i$. Thus, again by distributivity, we have $x=y$, contrary to the subdirect irreducibility of $L$.

Blyth and Varlet observed in [3] that, for any $L \in \mathbf{M S}, L^{00}=\left\{x \in L ; x=x^{00}\right\}$ is a de Morgan subalgebra of $L$, that the relation $\Phi$ defined $L$ by $x \equiv y(\Phi) \Leftrightarrow x^{00}=y^{00}$ is a congruence of $L$ and that $L^{00} \cong L / \Phi$. The same is obviously true for any $L \in \mathscr{K}_{1,1}$. Moreover, it is known (see [1], for example) that a de Morgan algebra $L$ is simple if $a=a^{0}$, whenever $a \in L \backslash\{0,1\}$. An easy consequence of this and lemma 5 is the following extension of a key theorem in [3].

THEOREM 6. If $L \in \mathscr{K}_{1,1}$ and $L$ is subdirectly irreducible, then $L^{\circ 0}$ is a simple de Morgan algebra.

Again, the following is the counterpart of a result proved in [3] for MS.

COROLLARY 7. Let $L \in \mathscr{K}_{1,1}$ be non-trivial. Then $L$ is subdirectly irreducible if and only if $\omega \leqslant \Phi<\iota$.

Proof. The interval $[\Phi, \iota]$ in $\operatorname{Con}(L)$, the congruence lattice of $L$, is isomorphic to $\operatorname{Con}(L / \Phi) \cong \operatorname{Con}\left(L^{00}\right) \cong 2$, since $L^{00}$ is simple and non-trivial. Therefore, $\Phi<i$. Moreover, if $\Phi \neq \omega$ then the interval $[\omega, \Phi]$ in $\operatorname{Con}(L)$ is Boolean, since it coincides with the corresponding interval in the lattice of lattice congruences of $L$, which is Boolean by virtue of the fact that $L$ is finite (see [2]).

The next corollary reduces the amount of tedious, case-by-case examination necessary for the determination of the subdirectly irreducible algebras in $\mathscr{K}_{1,1}$ via this approach. 
COROLlaRY 8. Let $L$ be a subdirectly irreducible algebra in $\mathscr{K}_{1,1}$. Then

(i) $|[a] \Phi| \leqslant 2$, for all $a \in L$.

(ii) $|L| \leqslant 8$ and $|L| \neq 7$.

Proof. Suppose that $\Phi \neq \omega$. If $L$ has a $\Phi$-class having more than two elements than this class contains a three element chain $x<y<z$, say. Clearly, $\omega<\theta_{\text {lat }}(x, y)=\theta(x, y) \leqslant \Phi$ so that $\theta_{\text {lat }}(x, y)=\Phi=\theta_{\text {lat }}(y, z)$, since $\omega<\Phi$, and therefore $\Phi=\theta_{\text {lat }}(x, y) \wedge \theta_{\text {lat }}(y, z)=\omega$. Thus, (i) holds. For (ii), first observe that $L \in \mathscr{K}_{1,1}$ implies $[a] \Phi=\left[a^{00}\right] \Phi$, for any $a \in L$. Hence, $L / \Phi=\left\{[x] \Phi ; x \in L^{00}\right\}$ is a disjoint covering of $L$ by sets each having cardinality at most two. Therefore, $|L| \leqslant 2\left|L^{00}\right|$. Now, the simple de Morgan algebras are precisely the algebras $T, B, S$ and $M$ depicted in $\S 3$. Consequently, $\left|L^{00}\right| \leqslant 4$ and so $|L| \leqslant 8$. Also, if $|L|=7$ then $\left|L^{00}\right|=4$, so that $L^{00} \cong M$ and $L$ contains a complementary pair $a, b \notin\{0,1\}$. Thus, as a lattice, $L$ has the non-trivial direct decomposition $L \cong(a] \times(b]$ which is absurd because 7 is prime.

We now have enough information at hand to produce systematically the subdirectly irreducibles $L$ in $\mathscr{K}_{1,1}$. The idea is to consider each of the possibilities for $L^{00}$ in turn and apply corollaries 7 and 8 to test for subdirect irreducibility. In summary, we have:

Case (i): $L^{00}$ is trivial. This produces only the trivial algebra $T$.

Case (ii): $L^{00}=\{0,1\}$. This produces $T, B, S, \check{S}$ and $S_{1}$.

Case (iii): $L^{00}=\{0, a, 1\}$, with $a=a^{0}$. First, note that a subdirectly irreducible algebra $L$ in this category has $3 \leqslant|L| \leqslant 6$. We deal with the possible chain algebras first. Those having at most four elements are precisely $K, K_{1}, K_{2}, \check{K}_{1}, \check{K}_{2}$ and none has either 5 or 6 elements, because at least two of the three $\Phi$-classes of such an algebra must contain exactly 2 elements but then it is easily seen that $\omega \$ \Phi$. Next, we deal with the subdirectly irreducible algebras in this category that are not chains. It is a simple, but tedious, exercise to show that there are no such algebras having four elements. The only five element algebras that can be produced are $K_{3}, \check{K}_{3}, L$ and $\check{L}$ while the six element ones are precisely $N$ and $\check{N}$.

Case (iv): $L^{00}=\{0, a, b, 1\}$, with $a=a^{0}, b=b^{0}$. Any subdirectly irreducible algebra $L$ in this category contains a complementary pair $a, b \notin\{0,1\}$ and so, as a lattice, has a non-trivial direct decomposition. Thus, the lattice reduct of $L$ is either $\mathbf{2} \times \mathbf{2}, \mathbf{3} \times \mathbf{2}, \mathbf{4} \times \mathbf{2}$ (where 2, 3 and 4 denote the 2, 3 and 4 element chains) or $2^{3}$. The first event yields only $M$, the second yields $M_{1}$ and $\check{M}_{1}$, the third produces none and the fourth yields only $B_{1}$.

\section{REFERENCES}

1. R. Balbes and Ph. Dwinger, Distributive Lattices (University of Missouri Press, 1974).

2. J. Berman, Distributive lattices with an additional unary operation, Aequationes Math. 16 (1977), 165-171.

3. T. S. Blyth and J. C. Varlet, On a common abstraction of de Morgan algebras and Stone algebras, Proc. Roy. Soc. Edinburgh. 94A (1983), 301-308.

4. S. Burris and H. P. Sankappanavar, A Course in Universal Algebra, (Springer-Verlag 1981). 
5. M. S. Goldberg, Distributive Ockham algebras: free algebras and injectivity, Bull. Austral. Math. Soc. 24 (1981), 161-203.

6. H. Priestley, Representation of distributive lattices by means of ordered Stone spaces, Bull. London Math. Soc. 2 (1970), 186-190.

7. H. Priestley, Ordered topological spaces and the representation of distributive lattices, Proc. London Math. Soc. (3), 24 (1972), 507-530.

8. A. Urquhart, Distributive lattices with a dual homomorphic operation, Studia Logica 38 (1979), 201-209.

Department of Mathematics,

UNIVERSITY OF GLASGOW,

SCOTLAND, U.K. 